\title{
Parkinson's Disease and Cancer
}

\section{Insights for Pathogenesis from Epidemiology}

\author{
Marco D'Amelio, ${ }^{a}$ Paolo Ragonese, ${ }^{a}$ Gabriella Sconzo, ${ }^{b}$ \\ Paolo Aridon, ${ }^{a}$ and Giovanni Savettieri ${ }^{a}$
}

\author{
${ }^{a}$ Dipartimento Universitario di Neuroscienze Cliniche, Universitá degli Studi di Palermo, \\ Palermo, Italy \\ ${ }^{b}$ Dipartiomento di Biologia Cellulare e dello Sviluppo, Universitá degli Studi di Palermo, \\ Palermo, Italy
}

\begin{abstract}
Epidemiological evidence suggests a reduced incidence of many common types of cancers in individuals with Parkinson's disease (PD). Parkinson's disease and cancer are two diseases that result from an excessive signaling by one of two forces driving cells to opposite directions. PD results from the excessive death of dopaminergic neurons in the substantia nigra pars compacta $(\mathrm{SNc})$ in the brain, while uncontrolled growth is the key property of cancer. Parkinson's disease is a complex disorder, probably due in most of the cases to the interaction of environment and genes. Many genes responsible for familial forms of PD are supposed to have a supportive role in regulating or maintaining the cell cycle, a fact that allows us to assume their interaction in tumorigenesis. Understanding the nature of these processes may help researchers find new and more efficacious therapeutic approaches for both diseases.
\end{abstract}

Key words: Parkinson's disease; cancer; epidemiology; genetics; risk factors

\section{Introduction}

Parkinson's disease (PD) is the second most common neurodegenerative disorder, after Alzheimer's disease. PD is associated with a selective loss of dopaminergic neurons in the nigrostriatal pathway of the brain, and pathologically it is characterized by the presence of Lewy bodies whose primary structural component is alpha-synuclein. Clinical manifestations include motor abnormalities (tremor, rigidity, slowness, balance problems), autonomic disturbances, and nonmotor symptoms (depression and cognitive impairment).

The cause of PD is still unknown. Only in a minority of cases is PD determined by major gene mutations, while in most cases, nongenetic

Address for correspondence: Giovanni Savettieri, M.D., Dipartimento Universitario di Neuroscienze Cliniche, Università degli Studi di Palermo, Via Gaetano La Loggia 1-90129 Palermo, Italy. Voice: +39-091-6555146; fax: +39-091-6555147.gsavetti@tin.it factors probably interacting with susceptibility genes play the most important role.

Many environmental risk factors for PD have been proposed on the basis of presumed pathogenetic mechanisms of the disease. The first evidence suggesting that PD might be the consequence of environmental toxin goes back to 1983, when several people developed a parkinsonian syndrome after intravenous injection of drugs contaminated with 1-methyl4-phenyl-1,2,3,6-tetra hydropyridine (MPTP). MPTP was successively demonstrated to selectively damage dopaminergic cells in the substantia nigra and therefore came the hypothesis that exposure to environmental toxins was possibly one of the causes of PD. ${ }^{1}$

Cigarette smoking and coffee consumption are among the most studied risk factors for PD. Consistent results across epidemiological studies have been obtained showing a reduced risk of PD among cigarette smokers and coffee drinkers. ${ }^{2}$ PD risk is increased in men 
compared to women, and a protective role of estrogens has been hypothesized. ${ }^{3-5}$ So far, however, literature investigating this topic is not yet definitive. Other factors have been investigated (antioxidants, fat and fatty acids, dietary iron) but results of the studies have been not yet convincing. ${ }^{6}$

In recent years, the discovery of several causative monogenetic mutations determined an increase in the interest of the scientific community in PD. These mutations, however, explain only a small proportion of all PD, while approximately $90 \%$ of cases are still considered sporadic. Therefore, the pathogenetic mechanisms underlying the selective dopaminergic cell loss in PD are still not understood. Mitochondrial dysfunction, oxidative stress, and protein mishandling seem to play a central role in PD pathogenesis, ${ }^{7}$ and these processes, in sporadic PD, might be induced by nongenetic factors, probably interacting with susceptibility genes. The need to search for nongenetic causes is still striking in order to further understand the pathogenesis of PD and develop effective therapeutic strategies.

In the last two decades it has increasingly been suggested that PD patients are in some way protected from cancer. Some epidemiological evidence suggests in fact a low incidence of many common types of cancers in individuals with PD.

In this article, we will first describe epidemiological studies supporting the inverse association between cancer and PD, and then we will focus on possible explanations for the inverse association.

\section{Cancer and Parkinson's Disease}

\section{Epidemiological Studies}

Evidence in favor of the inverse association between PD and cancer goes back to mid 1900 s, when it was pointed that "for reasons as yet unclear, cancer is phenomenally rare in paralysis agitans." ${ }^{\prime 8}$ Later a significant reduced risk of death for cancer among PD patients was reported. ${ }^{9}$

Recently epidemiologists approached the study of the association between cancer and PD in two different ways. Cancer and PD are chronic disorders with a long time interval between the onset of pathogenic alterations and clinical manifestations. As the association between PD and cancer might have several explanations, it is methodologically important to define, in order to estimate a cause-effect relationship, if cancer happens before or after PD onset. While in the case of onset of cancer before PD the best study design is a case-control study, a cohort design is the best method to estimate the incidence of cancer in previously diagnosed PD patients. Therefore, this review will gather results of studies stratified by those who looked at cancer occurrence before PD onset (Table 1) and those who estimated cancer incidence in PD patients (Table 2).

\section{Cancer Preceding PD Onset}

Only four studies looked at the frequency of cancer preceding PD onset. The first study was conducted in Rochester, Minnesota. ${ }^{10}$ This was a population-based case-control study performed using the medical records linkage system of the Rochester Epidemiology Project. One-hundred ninety-six PD incident cases during the period 1976-1995 were matched by age and gender to a general population control. Overall frequency of cancer was lower in cases $(19.4 \%)$ than in controls $(23.5 \%)$. The inverse association was stronger in women and in individuals younger than 71 years of age. Though none of the associations reached the statistical significance, bladder and breast cancer were less frequent among cases, while prostate cancer was more common among controls.

The second case-control study, chronologically, was based on prevalent PD cases. ${ }^{11}$ Starting from a sample of 368 individuals complaining of parkinsonian signs, the authors included in the study a sample of 222 PD individuals. In fact, excluded from the original sample were those individuals not satisfying PD criteria 


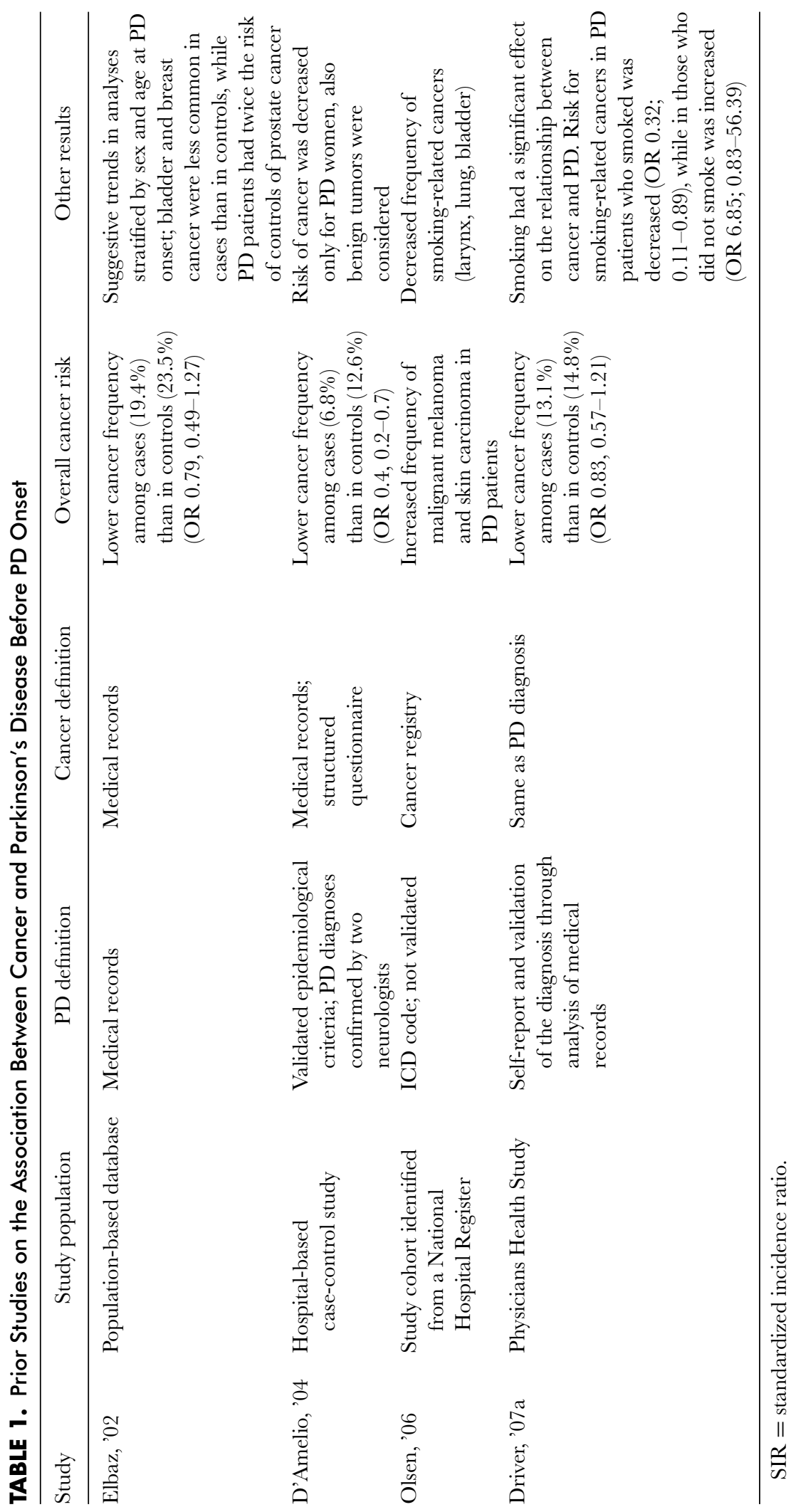




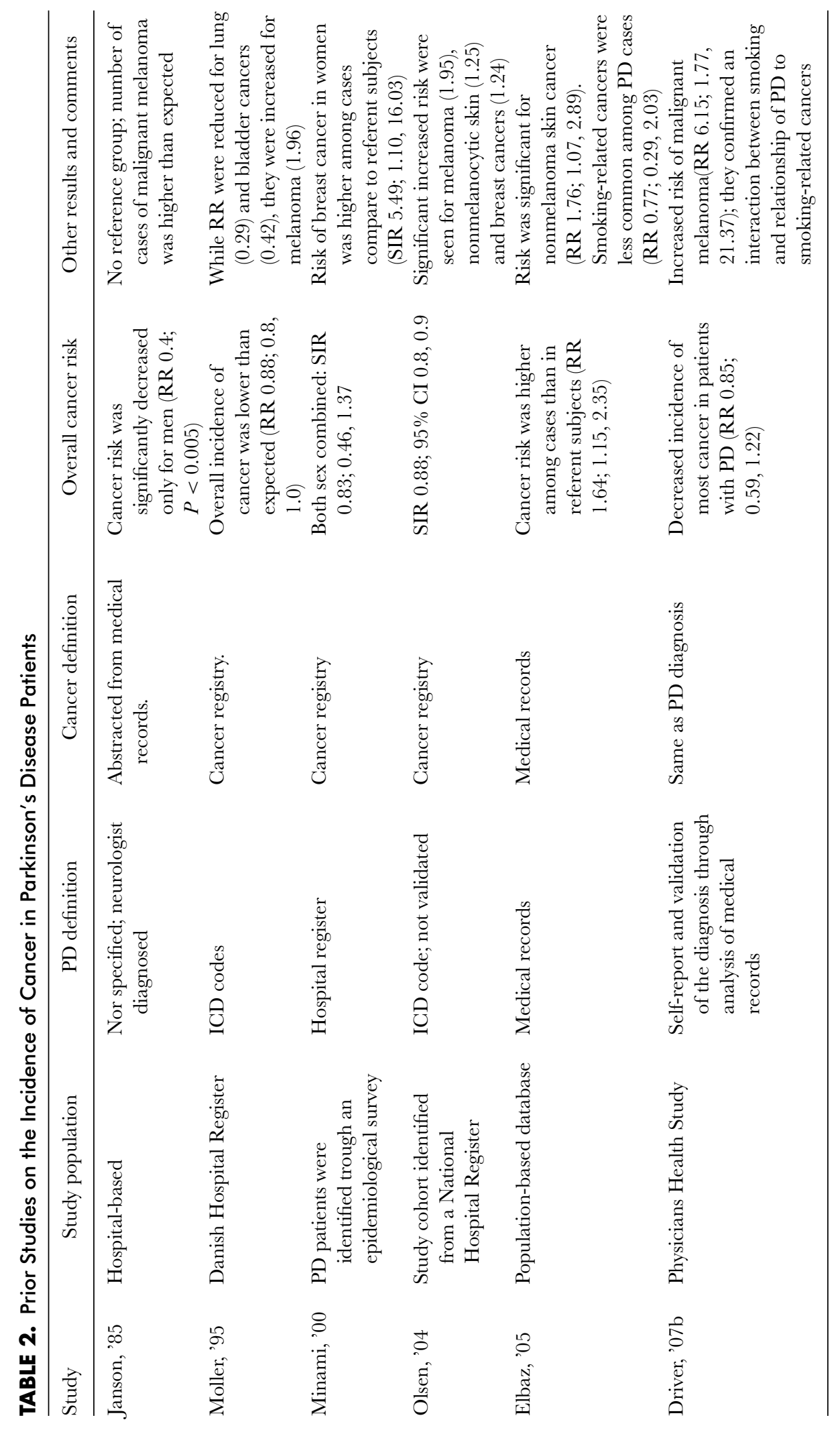


(110 patients), those with a Mini-Mental State Examination (MMSE) score lower than 24 (25 patients), and 11 persons refusing to participate in the study. Cases were then matched by age and gender to 222 PD free individuals. The frequency of cancer preceding PD onset was significantly lower among PD patients $(6.8 \%)$ compared to controls (12.6\%). Also, this study found a decreased significant risk of cancer only for women and, though based on small numbers, in spite of an overall reduced risk of cancer in PD patients, breast cancer was twice more common among cases than in controls.

The third study observed an increased significant prevalence of malignant melanoma and skin carcinoma prior to the first hospital contact for $\mathrm{PD},{ }^{12}$ with an overall odds ratios of 1.4 (95\% CI 1.0-2.0) and 1.3 (95\% CI 1.11.4), respectively. On the contrary, a reduced prevalence of cancers at smoking-related sites in patients before their first hospital contact for Parkinson's disease was observed. Findings of this study supported previous observation of an increased risk of melanoma prior to PD diagnosis observed by Elbaz et al. (OR 1.5; 95\% CI 0.3-9.0), weakening the hypothesis that skin cancers might be caused by PD treatment. For the authors the finding of a decreased prevalence of smoking-related cancers preceding Parkinson's disease was consistent with the wellknown higher risk of Parkinson's disease among nonsmokers.

Finally, the most recent study ${ }^{13}$ used data from the Physician's Health Study (PHS), enrolling 22,071 male physicians. The authors identified 487 PD incident cases and matched them to 487 controls. They then evaluated a history of cancer prior to the index date that was confirmed by medical record review. Also this study observed a decrease, though not significant, frequency of cancer of any type preceding the diagnosis of PD.

\section{Cancer after PD Onset}

Studies estimating cancer incidence in PD patients are more abundant. The first study used data from two separate surveys and calculated the expected incidence rates for malignancies in a sample of $406 \mathrm{PD}$ patients with PD. ${ }^{14}$

Cancer incidence was about one-third that of the general population. Relative risk of cancer increased after the onset of PD and after the treatment was started, but it was still half that of the general population. Thyroid cancer in females (3 cases) and melanomas (2 cases) were significantly more common in $\mathrm{PD}$ patients than expected.

Using three computerized registries in Denmark $^{15}$ a cohort of 7046 patients with PD, located from three computerized Danish registries, were matched to the Danish cancer registry and the Danish registry of deaths. Cancer incidence in PD patients (observed number of cases) was compared to the expected number of cancer cases. A significantly lower risk of cancer was observed for PD patients (relative risk [RR] 0.88). In particular, PD patients had a lower risk of smoking-related cancer (lung and bladder), while they showed a two-fold increased risk of malignant melanoma.

A prognosis study of 246 PD patients ${ }^{16}$ identified in an epidemiological survey on the number of patients treated for intractable neurological diseases reported a lower, though not significant, decreased risk of cancer in both genders. However, risk of breast cancer was 5.5 times higher in PD patients compared to the general population.

A significant increased risk of breast cancer was also observed in a Danish study, ${ }^{17}$ which observed also a twofold increased risk for malignant melanomas and a slight increased incidence of nonmelanocytic skin cancers. Overall, smoking-related cancers were less common in PD patients. Interestingly, as already reported by other authors, risk for melanoma skin cancer decreased gradually with increasing periods of follow-up, making less likely a role of levodopa treatment in melanoma development.

Incidence of cancer ${ }^{18}$ was ascertained in an historical cohort of PD patients. Risk of cancer was higher among PD patients than in 
the general population (RR 1.6; 95\% CI 1.22.4), but most cancer risk was attributable to melanoma skin cancer (RR 1.8; 95\% CI 1.11.9). The estimate of the effect of the cumulative dose of levodopa was specifically calculated and no evidence for a dose-effect relation was observed.

In the last study ${ }^{19} 487$ incident cases of PD without cancer preceding PD onset were identified and matched to PD-free individuals. Consistent with previous studies individuals with PD had a lower cancer risk. PD patients had in particular less lung (RR 0.3), colorectal (RR 0.54), and bladder (RR 0.68) malignancies. Among the studies described, this was the one with the highest significant risk for melanoma skin cancer (RR 6.15). However, smoking status significantly modified the relationship between PD and smoking-related cancers, suggesting a gene-environment interaction. PD patients who smoked were in fact at a reduced risk for smoking-related cancers (RR 0.33), whereas nonsmoker PD patients were at increased risk (RR 1.8).

\section{Discussion}

Case-control studies and large prospective studies reported a suggestive decreased frequency of smoking and nonsmoking related cancers among patients with PD. Though most cancers appear to be less common, a few cancer types, including melanomas, thyroid, and breast cancer, have been reported to occur with increased rates in PD patients.

Ultraviolet radiation (UVR) exposure and individual phenotype are well-known major etiologic risk factors for cutaneous melanoma. ${ }^{20}$ PD patients might have higher risk of melanoma skin cancer because of high solar exposure due to other risk factors for PD (i.e., rural living, farming). The association between Parkinson's disease and melanoma could be explained by the association between farming and/or rural living, both conditions gen- erally associated with higher solar exposure, and PD. However, it seems unlikely that PD patients are individuals who significantly spend more time than the general population in the sunlight.

Other possible explanations consider the common embryonic origin of melanocytes and neurons. Levodopa is a substrate for the synthesis of melanin, and though according to some authors increased rate of melanoma in PD patients is unrelated to levodopa treatment, ${ }^{21}$ one of the most recent hypotheses linked these malignancies to the levodopa in genetic-susceptible individuals, ${ }^{22}$ suggesting again a gene-environment interaction.

Breast cancer is also more frequent in PD patients than in the general population. Ascertainment bias must be considered, as individuals with $\mathrm{PD}$ are more likely to seek medical care and receive a diagnostic investigation than individuals without PD. This statement is also supported by the contrasting result of an inverse association between breast cancer and PD when tumors are diagnosed before PD onset. $^{10}$

While we could have doubts about the predisposition for PD patients to develop some cancers, we are also more prone to believe to the inverse association between malignancies and PD.

The risk that a tumor is underdiagnosed in PD patients is very low. On the contrary, as it has already been suggested, patients with PD are more likely to see a medical doctor and as a result they would have higher risk to be diagnosed with a second disease.

$\mathrm{PD}$ is due to neurodegeneration of dopaminergic neurons of the substantia nigra, and it is just the process leading to cell death that might explain the relationship between PD and cancer.

The discovery of gene mutations associated with familial parkinsonian disorders and understanding their role in cell survival and cell death may unravel the relationship between these two disorders. 
TABLE 3. Familial Forms of PD

\begin{tabular}{lccc}
\hline Type & Loci & Gene & Inheritance \\
\hline PARK1 & $4 \mathrm{q} 21-23$ & $\alpha$-synuclein & AD \\
PARK2 & $6 \mathrm{q} 25.20-27$ & parkin & AR \\
PARK3 & $2 \mathrm{p} 13$ & unknown & AD \\
PARK4 & $4 \mathrm{q} 21-23$ & $\alpha$-synuclein & AD \\
PARK5 & $4 \mathrm{p} 14$ & UCH-L1 & AD \\
PARK6 & $1 \mathrm{p} 35-36$ & PINK1 & AR \\
PARK7 & $1 \mathrm{p} 36$ & DJ-1 & AR \\
PARK8 & $12 \mathrm{p} 11.2-\mathrm{q} 13.1$ & LRRK2 & AD \\
PARK9 & $1 \mathrm{p} 36$ & ATP13A2 & AR \\
PARK10 & $1 \mathrm{p} 32$ & unknown & SP \\
PARK11 & $2 \mathrm{q} 36-37$ & unknown & AD \\
PARK12 & Xq21-25 & unknown & SP \\
PARK13 & $2 \mathrm{p} 13$ & Omi/HtrA2 & AD? \\
\hline
\end{tabular}

$\mathrm{AD}=$ autosomal dominant; $\mathrm{AR}=$ autosomal recessive; $\mathrm{S}=$ sporadic

\section{Familial Forms of Parkinson's Disease}

Thirteen chromosome loci (Table 3) linked to familial forms of PD have been until now identified. As PARK1 and PARK4 represent the same locus, the number of the familial forms is 12 .

PARK1- and PARK4-linked PD is an autosomal dominant one, caused by mutations of the alpha-synuclein gene (SNCA); PARK1 is caused by missense mutations and PARK4 by multiplications of SNCA.

Alpha-synuclein was found to be widely expressed both in a variety of brain tumors $^{23,24}$ such as medulloblastoma, neuroblastoma, pineoblastoma, and ganglioma, as well as in peripheral cancers, including ovarian and breast cancers. ${ }^{25}$ Recently, alpha-synucleinoverexpressing cells transfected to human osteosarcoma MG63 cell line ${ }^{26}$ exhibited distinct features of differentiated osteoblastic phenotype. In alpha-synuclein-overexpressing cells, proteasome and kinase $\mathrm{C}$ activity were significantly decreased, while activity of lysosome was upregulated. Taken together these results suggest that the stimulatory effect of alphasynuclein on tumor differentiation may be attributed to downregulation of proteasome, which is further modulated by alterations of various factors, such as protein kinase $\mathrm{C}$ signaling pathway and a autophagy-lysosomal degradation system. It has been hypothesized that PD-related molecules might converge to regulate the activity of ubiquitin proteasome system (UPS) in tumor differentiation.

Parkin (PARK2) is the most common cause of inherited PD, ${ }^{27-29}$ accounting for up to $49 \%$ of familial recessive early-onset PD cases. The parkin gene is characterized by a large 1.4$\mathrm{Mb}$ genomic structure covering more than $40 \%$ of the 6q25-q27 chromosomal region, ${ }^{30}$ which frequently undergoes deletions in a wide spectrum of human neoplasms, such as hepatocellular carcinoma, ${ }^{31}$ ovarian and breast cancer, ${ }^{32}$ and hematological neoplasm. ${ }^{33}$

The deregulation of the parkin gene, observed in various human cancers, suggest that the parkin gene is important in tumorigenesis.

In particular, parkin gene may play an important role in the development of mammary and ovarian tumors. ${ }^{34}$ In this study DNA from 20 malignant breast tumors, 20 ovarian tumors, and corresponding nontumor tissues were genotyped. The analysis revealed deletions at the 6q25-q27 locus in 55\% of cases analyzed. Subsequently, analysis of parkin gene expression in a variety of human cancers, including malignant ovarian and breast tumors, revealed the reduction of lack of transcript in approximately $70 \%$ of the samples examined.

The authors concluded suggesting that parkin is a strong candidate tumor suppressor gene (TSG), located at human chromosome 6q25-q27, and that its reduced expression and inactivation by hemizygous or homozygous deletion may play an important role in ovarian and breast carcinogenesis and other human tumors.

Later, the histological spectrum of tumors in which the candidate TSG parkin is genetically altered was expanded also to non-smallcell lung cancer. ${ }^{35}$ 
Recently, ${ }^{36}$ a parkin-/- mouse lacking exon 3 of the parkin gene showed hepatocyte proliferation and developed macroscopic hepatic tumors with the characteristics of hepatocellular carcinoma. Microarray analyses revealed that parkin deficiency caused the alteration of gene expression profiles in the liver. Among them, endogenous follistatin is commonly upregulated in both nontumorous and tumorous liver tissues of parkin-deficient mice. Parkin deficiency resulted in suppression of caspase activation and made hepatocytes resistant to apoptosis in a follistatindependent manner. These results suggested that parkin deficiency caused enhanced hepatocyte proliferation and resistance to apoptosis, resulting in hepatic tumor development. The finding that parkin-deficient mice are susceptible to hepatocarcinogenesis provided the evidence that parkin is indeed a tumor suppressor gene.

Although this finding suggests that parkin is a TSG, it is not clear whether mutations in the gene, found in patients with PARK2, results in increased risk of cancer.

PARK5-linked PD is an autosomal dominant PD. The disease gene was reported as ubiquitin carboxyl-terminal hydrolase-L1 (UCH-L1). ${ }^{37}$ UCH-L1 is an enzyme that cleaves carboxy-terminal peptide bond of polyubiquitine chains. Thus, UCH-Ll is an ubiquitin-recycling enzyme. UCH-Ll is a neuron-specific enzyme and is one of the most abundant proteins in the brain, but also presents in neuroendocrine cells in the lung.

Human lung cancers, in fact, frequently overexpress the ubiquitin carboxyterminal hydrolase UCHL1 ${ }^{38}$ Aberrant DNA methylation is associated with many types of human cancers. A microarray analysis for genes whose expression was induced by treatment of human colon cancer cells with a demethylating agent $^{39}$ showed an upregulation and overexpression of seven known genes. Among these was the UCHL1 gene. UCHL1 silencing was observed in 11 of 12 human colorectal cancer cell lines, and its methylation was detected in 8 of 17 primary colorectal cancers. Further, UCHL1 silencing was observed in 6 of 13 ovarian cancer cell lines, and its methylation was detected in 1 of 17 primary ovarian cancers. These results showed that UCHL1 is inactivated in human colorectal and ovarian cancers by its promoter methylation, suggesting that disturbance of cellular ubiquitin levels is present. Regarding the ubiquitin-proteasome system, to which UCHL1 belongs, its important roles in various cellular processes, such as cell cycle, apoptosis, and intracellular signaling, and its disturbances in cancer development are well recognized. ${ }^{40,41}$

PARK7-linked PD is another young onset PD. The disease gene was identified as DJ-1. ${ }^{42}$ The size of DJ-1 is $24 \mathrm{~kb}$ with 8 exons encoding a protein consisting of 189 amino acids. PARK7-linked PD is very rare, and the function of DJ-1 protein is not well known. DJ-1 is a cytoplasmic protein that can translocate into the mitochondria. It has a strong antioxidative property. ${ }^{43}$ Downregulation of endogenous DJ1 protein of neuronal cell line was reported to enhance the cell death induced by oxidative stress. ${ }^{44}$ DJ-1 protein expression is increased upon oxidative stress induced by paraquat. ${ }^{45}$ As nigral neurons are exposed to high oxidative stress owing to the presence of dopamine, DJ-1 may be act as a strong antioxidative protein.

DJ-1 has been identified as a novel suppressor of PTEN (phosphatase and tensin homolog, a a human gene that acts as a tumor suppressor gene. ${ }^{46,47}$ It seems to play a role in human tumorigenesis and also to be a useful prognostic marker for cancer. Breast cancer patients have elevated levels of serum DJ-1 and circulating anti-DJ-1 autoantibodies. ${ }^{48}$ Moreover it is increased in primary non-small-cell lung carcinoma samples. ${ }^{49}$

Mutations in the PTEN-induced putative kinase (PINK1, of the PARK6 locus) have been recently identified in individuals with $\mathrm{PD} .{ }^{50,51}$

PARK6-linked PD is another form of young onset autosomal recessive PD. PINK1 has eight exons and cDNA spans $1.8 \mathrm{~kb}$. It encodes a 
protein with 581 amino acids. The protein is ubiquitously expressed including brain and systemic organs. Interestingly, it is a mitochondrial protein located in the matrix and the intermembrane space.

PINK1 have been shown to regulate cell death and/or the cell cycle, and several lines of evidence imply that malfunction of a shared biochemical pathway may lead to PD or cancer. PINK 1 in fact encodes a kinase that is downregulated in the absence of PTEN. ${ }^{52}$

Tough premature - as the most common domain encoded by cancer genes is the protein-kinase domain ${ }^{53}$-mutations in the leucine-rich-repeat kinase 2 gene (LRRK2 of the PARK8 locus) recently identified in individuals with PD could also influence cell-cycle control. ${ }^{54}$ PARK8-linked PD is now believed to be the most common form of autosomal dominant familial PD. Function of LRRK2 is not well-known, but alterations of LRRK2 proteinreducing kinase activity corresponded to a reduced neuronal toxicity. ${ }^{55}$

\section{Conclusions}

In this review we summarized results of epidemiological studies showing an overall reduced risk of cancer in patients with Parkinson's disease compared to that of general population. Whereas the risk of most cancers is reduced in PD patients, melanomas and breast cancers seem to occur more frequently in the PD population compared to controls. Cancer risk reduction cannot be attributed solely to the well-known reduced smoking habit of PD patients, as not only do smoking-related cancers, but also nonsmoking-related cancers appear to be less frequent in PD patients.

The explanation of this peculiar finding might be related to the involvement of common genes in both the diseases. PD-linked genes influencing cell-cycle control and predisposing individuals either to develop Parkinson's disease or a specific cancer provide in fact a biological basis for results of epidemiological studies.
Health or disease states are determined for all individuals by interactions between genes and environment. How the environment modifies gene expression and how this can incline an individual to develop a disease needs to be explored in a productive way, one that considers exposure to known risk factors in genetically predisposed individuals. Future epidemiological studies will benefit from study designs that verify not only if specific types of cancers are more closely associated with PD, but in particular if they are associated with specific familial forms of PD. This will provide insights into the function of genes associated with Parkinson's disease, characterize biological pathways, and be important for the development of therapies directed to the cure of neurodegenerative diseases and malignancies.

\section{Conflicts of Interest}

The authors declare no conflicts of interest.

\section{References}

1. Langston, J.W., P. Ballard, J.W. Tetrud \& I. Irwin. 1983. Chronic parkinsonism in humans due to a product of meperidine-analog synthesis. Science 219: 979-980.

2. Hernán, M.A., B. Takkouche, F. Caamaño-Isorna \& J.J. Gestal-Otero. 2002. A meta-analysis of coffee drinking, cigarette smoking, and the risk of Parkinson's disease. Ann. Neurol. 52: 276-284.

3. Ragonese, P., M. D’Amelio \& G. Savettieri. 2006. Implications for estrogens in Parkinson's disease: An epidemiological approach. Ann. N. Y. Acad. Sci. 1089: 373-382.

4. Ragonese, P., M. D’Amelio, G. Callari, et al. 2006. Age at menopause predicts age at onset of Parkinson's disease. Mov. Disord. 21: 2211-2214.

5. Ragonese, P., M. D’Amelio, G. Salemi, et al. 2004. Risk of Parkinson disease in women: Effect of reproductive characteristics. Neurology 62: 2010-2014.

6. de Lau, L.M. \& M.M. Breteler. 2006. Epidemiology of Parkinson's disease. Lancet Neurol. 5: 525-535.

7. Greenamyre, J.T. \& T.G. Hastings. 2004. Biomedicine: Parkinson's - divergent causes, convergent mechanisms. Science 304: 1120-1122.

8. Doshay, L.J. 1954. Problem situations in paralysis agitans. FAMA 156: 680-684. 
9. Hoehn, M.M. \& M.D. Yahr. 1967. Parkinsonism: Onset, progression and mortality. Neurology 17: 427442.

10. Elbaz, A., B.J. Peterson, P. Yang, et al. 2002. Nonfatal cancer preceding Parkinson's disease: A case-control study. Epidemiology 13: 157-164.

11. D'Amelio, M., P. Ragonese, L. Morgante, et al. 2004. Tumor diagnosis preceding Parkinson's disease: A case-control study. Mov. Disord. 19: 807-811.

12. Olsen, J.H., S. Friis \& K. Frederiksen. 2006. Malignant melanoma and other types of cancer preceding Parkinson disease. Epidemiology 17: 582-587.

13. Driver, J.A., T. Kurth \& J.E. Buring. 2007a. Prospective case-control study of nonfatal cancer preceding the diagnosis of Parkinson's disease. Cancer Causes Control 18: 705-711.

14. Jansson, B. \& J. Jankovic. 1985. Low cancer rates among patients with Parkinson's disease. Ann. Neurol. 17: 505-509.

15. Møller, H., L. Mellemkjaer, J.K. McLaughlin, et al. 1995. Occurrence of different cancers in patients with Parkinson's disease. BM7 310: 1500-1501.

16. Minami, Y., R. Yamamoto, M. Nishikouri, et al. 2000. Mortality and cancer incidence in patients with Parkinson's disease. f. Neurol. 247: 429-434.

17. Olsen, J.H., S. Friis \& K. Frederiksen. 2005. Atypical cancer pattern in patients with Parkinson's disease. Br. F. Cancer 92: 201-205.

18. Elbaz, A., Peterson, B.J. Bower \& J.H. Yang. 2005. Risk of cancer after the diagnosis of Parkinson's disease: A historical cohort study. Mov. Disord. 20: 719725 .

19. Driver, J.A., G. Logroscino, J.E. Buring, et al. $2007 \mathrm{~b}$. A prospective cohort study of cancer incidence following the diagnosis of Parkinson's disease. Cancer Epidemiol. Biomarkers Prev. 16: 1260-1265.

20. Fortes, C. \& E. de Vries. 2008. Nonsolar occupational risk factors for cutaneous melanoma. Int. 7. Dermatol. 47: 319-328.

21. Olsen, J.H., K. Tangerud, L. Wermuth, et al. 2007. Treatment with levodopa and risk for malignant melanoma. Mov. Disord. 22: 1252-1257.

22. Zanetti, R., S. Rosso \& D.I. Loria. 2007. Parkinson's disease and cancer. Cancer Epidemiol. Biomarkers Prev. 16: 1081 .

23. Kawashima, M., S.O. Suzuki, K. Doh-ura, et al. 2000. Acta Neuropathol. (Berl.) 99: 154-160.

24. Fung, K.M., L.B. Rorke \& B. Giasson. 2003. Acta Neuropathol. (Berl.) 106: 167-175.

25. Bruening, W., B.I. Giasson \& A.J. Klein-Szanto. 2000. Synucleins are expressed in the majority of breast and ovarian carcinomas and in preneoplastic lesions of the ovary. Cancer 88: 2154-2163.

26. Fujita, M., S. Sugama, M. Nakai, et al. 2007. Alphasynuclein stimulates differentiation of osteosarcoma cells: Relevance to downregulation of proteasome activity. F. Biol. Chem. 282: 5736-5748.

27. Lucking, C.B., A. Durr, V. Bonifati, et al. 2000. Association between early-onset Parkinson's disease and mutations in the parkin gene. $\mathcal{N}$. Engl. F. Med. 342: 1560-1567.

28. Deng, H., W. Le, Y. Guo, C.B. Hunter, et al. 2006. Heterogeneous phenotype in a family with compound heterozygous parkin gene mutations. Arch. Neurol. 63: 273-277.

29. Deng, H., W.D. Le, C.B. Hunter, et al. 2007. A family with Parkinson's disease, essential tremor, Bell's palsy and parkin mutations. Arch. Neurol. 64: $421-$ 424.

30. Mizuno, Y., N. Hattori, S. Kubo, et al. 2008. Progress in the pathogenesis and genetics of Parkinson's disease. Philos. Trans. R. Soc. Lond. B Biol. Sci. 363: 22152227.

31. Wang, F., S. Denison, J.P. Lai, et al. 2004. Parkin gene alterations in hepatocellular carcinoma. Genes Chromosomes Cancer 40: 85-96.

32. Denison, S.R., F. Wang, N.A. Becker, et al. 2003. Alterations in the common fragile site gene parkin in ovarian and other cancers. Oncogene 22: 8370-8378.

33. Agirre, X., J. Román-Gómez, I. Vázquez, et al. 2006. Abnormal methylation of the common PARK2 and PACRG promoter is associated with downregulation of gene expression in acute lymphoblastic leukemia and chronic myeloid leukemia. Int. f. Cancer 118: 1945-1953.

34. Cesari, R., E.S. Martin, G.A. Calin, et al. 2003. Parkin, a gene implicated in autosomal recessive juvenile parkinsonism, is a candidate tumor suppressor gene on chromosome 6q25-q27. Proc. Natl. Acad. Sci. USA 100: 5956-5961.

35. Picchio, M.C., E.S. Martin, R. Cesari, et al. 2004. Alterations of the tumor suppressor gene parkin in non-small cell lung cancer. Clin. Cancer Res. 10: 2720 2724.

36. Fujiwara, M., H. Marusawa, H.Q. Wang, et al. 2008. Parkin as a tumor suppressor gene for hepatocellular carcinoma. Oncogene 27: 6002-6011.

37. Leroy, E., R. Boyer \& G. Auburger. 1998. The ubiquitin pathway in Parkinson's disease. Nature 395:451452.

38. Long, E.M. \& M.A. Long, et al. 2003. Stimulation of the murine Uchll gene promoter by the B-Myb transcription factor. Lung Cancer 42: 9-21.

39. Okochi-Takada, E., K. Nakazawa, M. Wakabayashi, et al. 2006. Silencing of the UCHLl gene in human colorectal and ovarian cancers. Int. F. Cancer 119: 1338-1344.

40. Mani, A. \& E.P. Gelmann. 2005. The ubiquitinproteasome pathway and its role in cancer. 7 . Clin. Oncol. 23: 4776-4789. 
41. Zhang, H.G., J. Wang, X. Yang, et al. 2004. Regulation of apoptosis proteins in cancer cells by ubiquitin. Oncogene 23: 2009-2015.

42. Bonifati, V. et al. 2003. Mutations in the DJ-1 gene associated with autosomal recessive early-onset parkinsonism. Science 299: 256-259.

43. Moore, D.J., L. Zhang, J. Troncoso, et al. 2005. Association of DJ-1 and parkin mediated by pathogenic DJ-1 mutations and oxidative stress. Hum. Mol. Genet. 14: $71-84$.

44. Yokota, T., K. Sugawara, K. Ito, et al. 2003. Down regulation of DJ-1 enhances cell death by oxidative stress, ER stress, and proteasome inhibition. Biochem. Biophys. Res. Commun. 312: 1342-1348.

45. Mitsumoto, A., Y. Nakagawa, A. Takeuchi, et al. 2001. Oxidized forms of peroxiredoxins and DJ-1 on two-dimensional gels increased in response to sublethal levels of paraquat. Free Radic. Res. 35: 301310.

46. Kim, R.H., M. Peters, Y. Jang, et al. 2005. DJ-1, a novel regulator of the tumor suppressor PTEN. Cancer Cell 7: 263-273.

47. Kim, R.H. \& T.W. Mak. 2006. Tumours and tremors: How PTEN regulation underlies both. Br. F. Cancer 94: $620-624$.

48. Le Naour, F., D.E. Misek, M.C. Krause, et al. 2001.
Proteomics-based identification of RS/DJ-1 as a novel circulating tumor antigen in breast cancer. Clin. Cancer Res. 7: 3328-3335.

49. MacKeigan, J.P., C.M. Clements, J.D. Lich, et al. 2003. Proteomic profiling drug-induced apoptosis in non-small cell lung carcinoma: Identification of RS/DJ-1 and RhoGDIalpha. Cancer Res. 63: 69286934.

50. Valente, E.M. et al. 2004a. Hereditary early-onset Parkinson's disease caused by mutations in PINK1. Science 304: 1158-1160.

51. Valente, E.M. et al. 2004b. PINK1 mutations are associated with sporadic early-onset Parkinsonism. Ann. Neurol. 56: 336-341.

52. West, A.B., V.L. Dawson \& T.M. Dawson. 2005. To die or grow: Parkinson's disease and cancer. Trends Neurosci. 28: 348-352.

53. Futreal, P.A., L. Coin, M. Marshall, et al. 2004. A census of human cancer genes. Nat. Rev. Cancer 4: 177-183.

54. Paisan-Ruiz, C. et al. 2004. Cloning of the gene containing mutations that cause PARK8-linked Parkinson's disease. Neuron 44: 595-600.

55. Smith, W.W., Z. Pei, H. Jiang, et al. 2006. Kinase activity of mutant LRRK2 mediates neuronal toxicity. Nat. Neurosci. 9: 1231-1233. 